Pacific

Journal of

Mathematics

THE MÖBIUS CHARACTERIZATIONS OF WILLMORE TORI AND VERONESE SUBMANIFOLDS IN THE UNIT SPHERE

Zhen GuO, HAIZHONG Li AND ChangPing WANG

Volume $241 \quad$ No. 2

June 2009 


\title{
THE MÖBIUS CHARACTERIZATIONS OF WILLMORE TORI AND VERONESE SUBMANIFOLDS IN THE UNIT SPHERE
}

\author{
Zhen GuO, HAIZHONG Li AND ChANGPING WANG
}

\begin{abstract}
Suppose $M$ is a $\boldsymbol{m}$-dimensional submanifold without umbilic points in the $(m+p)$-dimensional unit sphere $S^{m+p}$. Four basic invariants of $M^{m}$ under the Möbius transformation group of $S^{m+p}$ are a symmetric positive definite 2-form $g$ called the Möbius metric, a section $B$ of the normal bundle called the Möbius second fundamental form, a 1-form $\Phi$ called the Möbius form, and a symmetric $(0,2)$ tensor $A$ called the Blaschke tensor. In the Möbius geometry of submanifolds, the most important examples of Möbius minimal submanifolds (also called Willmore submanifolds) are Willmore tori and Veronese submanifolds. In this paper, several fundamental inequalities of the Möbius geometry of submanifolds are established and the Möbius characterizations of Willmore tori and Veronese submanifolds are presented by using Möbius invariants.
\end{abstract}

\section{Introduction}

Let $x: M \rightarrow S^{m+p}$ be a $m$-dimensional submanifold without umbilic point in a $(m+p)$-dimensional unit sphere $S^{m+p}$. Let $\left\{e_{i}\right\}$ be a local orthonormal tangent frame field of $x$ for the standard metric $I=d x \cdot d x$ with the dual frame field $\left\{\theta_{i}\right\}$, let $\left\{e_{\alpha}\right\}$ be the local orthonormal normal frame field of $x$, let $I I=\sum_{i, j, \alpha} h_{i j}^{\alpha} \theta_{i} \theta_{j} e_{\alpha}$ be the second fundamental form, and let $\boldsymbol{H}=\sum_{\alpha} H^{\alpha} e_{\alpha}$ be the mean curvature vector of $x$. Let $\rho^{2}=(m /(m-1))|I I-(1 / m) \operatorname{tr}(I I) I|^{2}$. Then the positive definite 2-form $\boldsymbol{g}=\rho^{2} I$ is invariant under the Möbius transformations of $S^{m+p}$ and is called the Möbius metric of $x$. In [Wang 1998], the author gave the structure equations for Möbius geometry of submanifolds in the unit sphere. Three fundamental forms $\boldsymbol{\Phi}, \boldsymbol{A}$ and $\boldsymbol{B}$ appear naturally in the structure equations; together with $\boldsymbol{g}$, these determine the submanifold up to Möbius transformations of $S^{m+p}$. $\boldsymbol{\Phi}=\sum_{\alpha, i} C_{i}^{\alpha} \theta_{i} e_{\alpha}$ is called the Möbius form, $\boldsymbol{A}=\rho^{2} \sum_{i, j} A_{i j} \theta_{i} \theta_{j}$ the Blaschke

MSC2000: primary 53A30; secondary 53B25.

Keywords: Willmore tori, Veronese submanifolds, Möbius geometry of submanifolds.

Guo is supported by project numbers 10561010 and 10861013 of NSFC. Li is supported by Zhongdian grant number 10531090 of NSFC, and Wang is supported by the Jiechu grant of NSFC. 
tensor, and $\boldsymbol{B}=\rho B_{i j}^{\alpha} \theta_{i} \theta_{j} e_{\alpha}$ the Möbius second fundamental form. The relations among $\boldsymbol{\Phi}, \boldsymbol{A}$, and $\boldsymbol{B}$ and Euclidean invariants of $x$ are given by [Wang 1998]

$$
\begin{aligned}
& C_{i}^{\alpha}=-\rho^{-2}\left(H_{, i}^{\alpha}+\sum_{j}\left(h_{i j}^{\alpha}-H^{\alpha} \delta_{i j}\right) e_{j}(\log \rho)\right), \\
& A_{i j}=-\rho^{-2}\left(\operatorname{Hess}_{i j}(\log \rho)-e_{i}(\log \rho) e_{j}(\log \rho)-\sum_{\alpha} H^{\alpha} h_{i j}^{\alpha}\right) \\
& \quad-\frac{1}{2} \rho^{-2}\left(\sum_{i}\left((\log \rho)_{i}\right)^{2}-1+|\boldsymbol{H}|^{2}\right) \delta_{i j},
\end{aligned}
$$

where $\mathrm{Hess}_{i j}$ and $H_{, i}^{\alpha}$ are the Hessian matrix of $d x \cdot d x$ and the covariant derivative of the mean curvature vector field of $x$ in the normal bundle $N(M)$. Let

$$
\|\boldsymbol{A}\|^{2}=\sum_{i, j}\left(A_{i j}\right)^{2} \quad \text { and } \quad\|\boldsymbol{\Phi}\|^{2}=\sum_{\alpha, i}\left(C_{i}^{\alpha}\right)^{2} .
$$

Then both $\|\boldsymbol{A}\|^{2}$ and $\|\boldsymbol{\Phi}\|^{2}$ are Möbius invariants.

In the Euclidean geometry of submanifolds in the unit sphere, a famous result is this rigidity theorem of minimal submanifolds: If $x$ is a minimal immersion and the second fundamental form satisfies $|I I|^{2} \leq m /(2-1 / p)$, then either $|I I|^{2}=0$ or $|I I|^{2}=m /(2-1 / p)$ and $x$ is Euclidean equivalent to the geodesic sphere, a Clifford torus or a Veronese surface; see [Chern et al. 1970; Lawson 1969; Simons 1968]. Recently, Li [2001; 2002] modified this result for the case of a Willmore submanifold $x$ (satisfying the Euler-Lagrange equation of the Willmore functional). He proved that if $x$ satisfies $\rho^{2} \leq m /(2-1 / p)$, then $\rho^{2}=m /(2-1 / p)$ and $x$ is Euclidean equivalent to a Willmore torus or a Veronese surface. However, $\rho^{2}$ is not conformally invariant under the Möbius group of $S^{m+p}$. A natural question is, Do conformal invariants characterize Willmore tori and Veronese submanifolds in Möbius geometry? In this paper, we give a positive answer to this question. It should be noted that we don't need the assumption of Willmore submanifold for our theorems here. To state our results, we first introduce traceless Blaschke tensor $\tilde{\boldsymbol{A}}=\rho^{2} \sum_{i, j} \tilde{A}_{i j} \theta_{i} \theta_{j}$ and traceless tensor $\breve{\boldsymbol{B}}=\rho^{2} \sum_{i, j} \check{B}_{i j} \theta_{i} \theta_{j}$, where

$$
\tilde{A}_{i j}=A_{i j}-\frac{1}{m}\left(\sum_{k} A_{k k}\right) \delta_{i j} \quad \text { and } \quad \check{B}_{i j}=\frac{m-1}{m^{2}} \delta_{i j}-\sum_{k, \alpha} B_{i k}^{\alpha} B_{k j}^{\alpha} .
$$

We let $\|\tilde{\boldsymbol{A}}\|^{2}=\sum_{i, j}\left(\tilde{A}_{i j}\right)^{2}$. We denote by $\kappa$ the normalized Möbius scalar curvature of $x: M \rightarrow S^{m+p}$ (see (2-8)).

In the Möbius geometry of submanifolds, Willmore tori

$$
\left\{W_{k}^{n}=S^{k}(\sqrt{(m-k) / m}) \times S^{m-k}(\sqrt{k / m}), 1 \leq k \leq m-1\right\},
$$

which can also be obtained by exchanging the radii in the Clifford tori

$$
M_{k}^{n}=S^{k}(\sqrt{k / m}) \times S^{m-k}(\sqrt{(m-k) / m}),
$$


are the chief examples of stable Willmore submanifolds; see [Guo et al. 2001; Li 2001; 2002]. The main purpose of this paper is to obtain some geometric inequalities expressed in Möbius invariants, which then characterize Willmore tori and Veronese submanifolds when equalities hold in the inequalities. The main results are as follows.

Theorem 1.1. Let $x: M \rightarrow S^{m+p}$ be an m-dimensional compact submanifold in the unit sphere $S^{m+p}$. Then

$\int_{M}\left(\kappa-m\|\boldsymbol{\Phi}\|^{2}-\frac{m}{4(m-1)}\|\tilde{\boldsymbol{A}}+\check{\boldsymbol{B}}\|^{2}-\frac{1}{m^{2}}\left(m-2+\frac{1}{2}(m-1) \operatorname{sgn}(p-1)\right)\right) d M \leq 0$.

In particular, if

$$
\kappa-m\|\boldsymbol{\Phi}\|^{2}-\frac{m}{4(m-1)}\|\tilde{\boldsymbol{A}}+\check{\boldsymbol{B}}\|^{2} \geq \frac{1}{m^{2}}\left(m-2+\frac{m-1}{2} \operatorname{sgn}(p-1)\right),
$$

then $M$ is Möbius equivalent to either

(i) a Willmore torus $W_{k}^{m}=S^{k}(\sqrt{(m-k / m)}) \times S^{m-k}(\sqrt{k / m})$ in $S^{m+1}$, or

(ii) a Veronese surface in $S^{4}$.

Theorem 1.2. Let $x: M \rightarrow S^{m+p}$ be an m-dimensional compact submanifold in the unit sphere $S^{m+p}$. Let $K(x)$ be the function that assigns to each point of $M$ the infinimum of the Möbius sectional curvatures of $M$ at that point. Then

$$
\int_{M}\left(2(m+1) K-m \kappa-1 / m-m(m+2)\|\boldsymbol{\Phi}\|^{2}-m\|\tilde{\boldsymbol{A}}\|\right) d M \leq 0 .
$$

In particular, if

$$
2(m+1) K-m \kappa-m(m+2)\|\boldsymbol{\Phi}\|^{2}-m\|\tilde{\boldsymbol{A}}\| \geq 1 / m,
$$

then $1 / K=m(m+2)$ and $M$ is Möbius equivalent to the Veronese submanifold $S^{m}(\sqrt{2(m+1) / m})$.

Corollary 1.3. Let $x: M^{m} \rightarrow S^{m+p}$ be an m-dimensional compact submanifold with constant Möbius sectional curvature $c$ and vanishing Möbius form. Then

$$
c \leq \frac{1}{m(m+2)}
$$

with equality holding if and only if $M$ is Möbius equivalent to the Veronese submanifold $S^{m}(\sqrt{2(m+1) / m})$.

Remark 1.4. The functions on the right side of inequalities (1-5) and (1-6) are Möbius invariants. 
Remark 1.5. For related results about Möbius submanifolds in an unit sphere, see [Akivis and Goldberg 1996; 1997; Guo and Guo 2006; Hu and Li 2003; 2004; Li and Wang 2003a; 2003b; Li et al. 2001].

In addition, in Section 4 we prove two Möbius sectional curvature pinching results, Theorem 4.1 and Theorem 4.2.

\section{Möbius invariants for submanifolds in $S^{m+p}$}

In this section we define the Möbius invariants for submanifolds in $S^{m+p}$. For more detail we refer to [Wang 1998] or [Guo et al. 2001].

Let $R_{1}^{m+p+2}$ be the Lorentzian space with inner product

$$
\langle x, y\rangle=-x_{0} y_{0}+x_{1} y_{1}+\cdots+x_{m+p+1} y_{m+p+1},
$$

where $x=\left(x_{0}, x_{1}, \ldots, x_{m+p+1}\right)$ and $y=\left(y_{0}, y_{1}, \ldots, y_{m+p+1}\right)$. Let $x: M \rightarrow S^{m+p}$ be a submanifold of $S^{m+p}$ without umbilic points. The second fundamental form and the mean curvature vector are denoted by $I I$ and $\boldsymbol{H}$ respectively. Define the positive function $\rho=\sqrt{(m-1) / m}|I I-\boldsymbol{H} I|$, and define the Möbius position vector $Y: M \rightarrow R_{1}^{m+p+2}$ of $x$ by $Y=\rho(1, x)$.

Theorem 2.1 [Wang 1998]. Two submanifolds $x, \tilde{x}: M \rightarrow S^{m+p}$ are Möbius equivalent if and only if there exists a $T$ in the Lorentz group $O(m+p+2,1)$ on $R_{1}^{m+p+2}$ such that $Y=\tilde{Y} T$.

Since the Möbius group in $S^{m+p}$ is isomorphic to the subgroup $O^{+}(m+p+2,1)$ of $O(m+p+2,1)$, which preserves the positive part of the light cone in $R_{1}^{m+p+2}$, we know from Theorem 2.1 that the 2-form

$$
\boldsymbol{g}=\langle d Y, d Y\rangle=\rho^{2} d x \cdot d x
$$

is a Möbius invariant; see [Blaschke 1929; Chen 1984; Pedit and Willmore 1988; Wang 1998]. We call $\boldsymbol{g}$ the Möbius metric or the Möbius first fundamental form induced by $x$. Let $\Delta$ denote the Laplacian of $g$. Then $\langle\Delta Y, \Delta Y\rangle=1+m^{2} \kappa$, where $\kappa$ is the normalized scalar curvature of $g$. By defining

$$
N=-\frac{1}{m} \Delta Y-\frac{1}{2 m^{2}}\left(1+m^{2} \kappa\right) Y,
$$

we have

$$
\langle Y, Y\rangle=\langle N, N\rangle=0 \quad \text { and } \quad\langle Y, N\rangle=1 .
$$

Moreover, if we take a local orthonormal basis $\left\{E_{i}\right\}$ with respect to $g$ with dual basis $\left\{\omega_{i}\right\}$ and let $Y_{i}:=E_{i}(Y)$, then

$$
\left\langle Y_{i}, Y_{j}\right\rangle=\delta_{i j} \quad \text { and } \quad\left\langle Y_{i}, Y\right\rangle=\left\langle Y_{i}, N\right\rangle=0 \quad \text { for } 1 \leq i, j \leq m .
$$


Let $V$ be the orthogonal complement of $\operatorname{span}\left\{Y, N, Y_{i}\right\}$ in $R_{1}^{m+p+2}$. Then $V$ is a spacelike subspace of $R_{1}^{m+p+2}$, and we have the orthogonal decomposition

$$
R_{1}^{m+p+2}=\operatorname{span}\{Y, N\} \oplus \operatorname{span}\left\{Y_{1}, \ldots, Y_{m}\right\} \oplus V .
$$

Let $\left\{E_{\alpha}: m+1 \leq \alpha \leq m+p\right\}$ be an orthogonal basis of $V$. Then

$$
\left\{Y, N, Y_{1}, \ldots, Y_{m}, E_{m+1}, \ldots, E_{m+p}\right\}
$$

forms a moving frame in $R_{1}^{m+p+2}$ along $M$. For ranges of indices, we use the conventions $1 \leq i, j, k, \ldots \leq m$ and $m+1 \leq \alpha, \beta, \ldots \leq m+p$, and we sum each repeated index over its respective range unless it also appears unrepeated in the same context. Then the structure equations are given by

$$
\begin{aligned}
d Y & =\omega_{i} Y_{i}, \\
d N & =\psi_{i} Y_{i}+\phi_{\alpha} E_{\alpha}, \\
d Y_{i} & =-\psi_{i} Y-\omega_{i} N+\omega_{i j} Y_{j}+\omega_{i \alpha} E_{\alpha}, \\
d E_{\alpha} & =-\phi_{\alpha} Y-\omega_{i \alpha} Y_{i}+\omega_{\alpha \beta} E_{\beta},
\end{aligned}
$$

where $\left\{\psi_{i}, \omega_{i j}, \omega_{i \alpha}, \phi_{\alpha}, \omega_{\alpha \beta}\right\}$ are 1-forms on $M$ satisfying $\omega_{i j}=-\omega_{j i}, \omega_{i \alpha}=-\omega_{\alpha i}$ and $\omega_{\alpha \beta}=-\omega_{\beta \alpha}$. Let

$$
\psi_{i}=A_{i j} \omega_{j}, \quad \omega_{i \alpha}=B_{i j}^{\alpha} \omega_{j}, \quad \phi_{\alpha}=C_{i}^{\alpha} \omega_{i} .
$$

It is clear that $\boldsymbol{A}=A_{i j} \omega_{i} \otimes \omega_{j}, \boldsymbol{B}=B_{i j}^{\alpha} \omega_{i} \otimes \omega_{j} E_{\alpha}$, and $\boldsymbol{\Phi}=C_{i}^{\alpha} \omega_{i} E_{\alpha}$ are Möbius invariants; these are called the Blaschke tensor, the Möbius second fundamental form, and the Möbius form, respectively.

Remark 2.2. The relations among $\boldsymbol{A}, \boldsymbol{B}, \boldsymbol{\Phi}$ and the Euclidean invariants of $x$ are given by (1-1), (1-2) and (1-3).

Taking the exterior derivations of structure equations, we obtain the integrability conditions for the structure equations as follows:

$$
\begin{aligned}
A_{i j} & =A_{j i}, \quad B_{i j}^{\alpha}=B_{j i}^{\alpha} \\
A_{i j, k}-A_{i k, j} & =\left(B_{i k}^{\alpha} C_{j}^{\alpha}-B_{i j}^{\alpha} C_{k}^{\alpha}\right) \\
C_{i, j}^{\alpha}-C_{j, i}^{\alpha} & =\left(B_{i k}^{\alpha} A_{k j}-B_{j k}^{\alpha} A_{k i}\right), \\
B_{i j, k}^{\alpha}-B_{i k, j}^{\alpha} & =\delta_{i j} C_{k}^{\alpha}-\delta_{i k} C_{j}^{\alpha} \\
R_{i j k l} & =\left(B_{i k}^{\alpha} B_{j l}^{\alpha}-B_{i l}^{\alpha} B_{j k}^{\alpha}\right)+\left(\delta_{i k} A_{j l}+\delta_{j l} A_{i k}-\delta_{i l} A_{j k}-\delta_{j k} A_{i l}\right), \\
R_{\alpha \beta i j} & =\left(B_{i k}^{\alpha} B_{k j}^{\beta}-B_{j k}^{\alpha} B_{k i}^{\beta}\right), \\
R_{i j} & =-B_{i k}^{\alpha} B_{k j}^{\alpha}+\operatorname{tr}(A) \delta_{i j}+(m-2) A_{i j}, \\
\operatorname{tr}(A) & =\frac{1}{2 m}\left(1+m^{2} \kappa\right), \quad \sum_{i} B_{i i}^{\alpha}=0, \quad B_{i j}^{\alpha} B_{i j}^{\alpha}=(m-1) / m,
\end{aligned}
$$


where $R_{i j k l}$ and $R_{\alpha \beta i j}$ denote the curvature tensor of $\boldsymbol{g}$ and the normal curvature tensor of the normal connection, respectively. We let $\kappa=1 /(m(m-1)) \sum_{i, j} R_{i j i j}$ be the normalized scalar curvature of $x: M \rightarrow S^{m+p}$. The first covariant derivatives of $A_{i j}$ and $B_{i j}^{\alpha}$ are defined through

$$
\begin{aligned}
& A_{i j, k} \omega_{k}=d A_{i j}+A_{k j} \omega_{k i}+A_{i k} \omega_{k j}, \\
& B_{i j, k}^{\alpha} \omega_{k}=d B_{i j}^{\alpha}+B_{k j}^{\alpha} \omega_{k i}+B_{i k}^{\alpha} \omega_{k j}+B_{i j}^{\beta} \omega_{\beta \alpha},
\end{aligned}
$$

From (2-4) and (2-8) we have

$$
B_{i j, i}^{\alpha}=-(m-1) C_{j}^{\alpha} .
$$

The second covariant derivative of $A_{i j}$ and $B_{i j}^{\alpha}$ are defined through

$$
\begin{aligned}
& A_{i j, k l} \omega_{l}=d A_{i j, k}+A_{l j, k} \omega_{l i}+A_{i l, k} \omega_{l j}+A_{i j, l} \omega_{l k}, \\
& B_{i j, k l}^{\alpha} \omega_{l}=d B_{i j, k}^{\alpha}+B_{l j, k}^{\alpha} \omega_{l i}+B_{i l, k}^{\alpha} \omega_{l j}+B_{i j, l}^{\alpha} \omega_{l k}+B_{i j, k}^{\beta} \omega_{\beta \alpha} .
\end{aligned}
$$

We have the Ricci identities

$$
\begin{aligned}
& A_{i j, k l}-A_{i j, l k}=A_{t j} R_{t i k l}+A_{i t} R_{t j k l}, \\
& B_{i j, k l}^{\alpha}-B_{i j, l k}^{\alpha}=B_{t j}^{\alpha} R_{t i k l}+B_{i t}^{\alpha} R_{t j k l}+B_{i j}^{\beta} R_{\beta \alpha k l} .
\end{aligned}
$$

\section{The proofs of Theorems 1.1 and 1.2}

From the definition of the Laplacian, by using (2-8), (2-4), (2-13) and (2-11) we have

$$
\begin{aligned}
0= & \frac{1}{2} \Delta\left(B_{i j}^{\alpha} B_{i j}^{\alpha}\right)=B_{i j, k}^{\alpha} B_{i j, k}^{\alpha}+B_{i j}^{\alpha} B_{i j, k k}^{\alpha} \\
=\|\nabla \boldsymbol{B}\|^{2}+B_{i j}^{\alpha}\left(B_{l k}^{\alpha} R_{l i j k}+B_{i l}^{\alpha} R_{l k j k}\right. & \left.+B_{i k}^{\beta} R_{\beta \alpha j k}\right) \\
& -m\left(B_{i j}^{\alpha} C_{i}^{\alpha}\right)_{j}-m(m-1)\|\boldsymbol{\Phi}\|^{2} .
\end{aligned}
$$

Integrating this over $M$ gives a lemma:

Lemma 3.1. Suppose $M$ is an m-dimensional compact submanifold in the unit sphere $S^{m+p}$. Then we have

$$
\begin{aligned}
0=\int_{M}\left(\|\nabla \boldsymbol{B}\|^{2}-m(m-1) \|\right. & \boldsymbol{\Phi} \|^{2} \\
& \left.+B_{i j}^{\alpha}\left(B_{l k}^{\alpha} R_{l i j k}+B_{i l}^{\alpha} R_{l k j k}+B_{i k}^{\beta} R_{\beta \alpha j k}\right)\right) d M .
\end{aligned}
$$

By using (2-5), (2-6) and (2-7), we have the calculation

$$
\begin{aligned}
B_{i j}^{\alpha}\left(B_{l k}^{\alpha} R_{l i j k}\right. & \left.+B_{i l}^{\alpha} R_{l k j k}+B_{i k}^{\beta} R_{\beta \alpha j k}\right) \\
=- & \operatorname{tr}\left(\left(B^{\alpha} B^{\beta}-B^{\beta} B^{\alpha}\right)\left(B^{\beta} B^{\alpha}-B^{\alpha} B^{\beta}\right)\right) \\
& \quad\left(\operatorname{tr} B^{\alpha} B^{\beta}\right)\left(\operatorname{tr} B^{\alpha} B^{\beta}\right)+((m-1) / m) \operatorname{tr}(A)+m \operatorname{tr}\left(B^{\alpha} B^{\alpha} A\right),
\end{aligned}
$$


where $B^{\alpha}$ denotes the matrix $\left(B_{i j}^{\alpha}\right)$. Since at least one element of $B^{\alpha}$ is nonzero, we have the following lemma, which is due to Li A.-M. and Li J.-M. [1992] for $p \geq 2$, and which is obvious for $p=1$.

Lemma 3.2. We have

$$
\begin{aligned}
\operatorname{tr}\left(\left(B^{\alpha} B^{\beta}-B^{\beta} B^{\alpha}\right)\left(B^{\beta} B^{\alpha}-B^{\alpha} B^{\beta}\right)\right)+\left(\operatorname{tr} B^{\alpha} B^{\beta}\right)\left(\operatorname{tr} B^{\alpha} B^{\beta}\right) \\
\leq\left(1+\frac{1}{2} \operatorname{sgn}(p-1)\right)\|\boldsymbol{B}\|^{4}
\end{aligned}
$$

with equality holding if and only if either

(i) $p=1$, or

(ii) $p=2$ and $B^{m+1}$ and $B^{m+2}$ can be transformed simultaneously by an orthogonal matrix into $\lambda \tilde{B}^{n+1}$ and $\mu \tilde{B}^{n+2}$, respectively, where

$$
\begin{array}{ll}
\tilde{B}_{12}^{m+1}=\tilde{B}_{21}^{m+1}=1 & \text { and } \tilde{B}_{i j}^{m+1}=0 \text { otherwise, } \\
\tilde{B}_{11}^{m+2}=-\tilde{B}_{22}^{m+2}=1 & \text { and } \tilde{B}_{i j}^{m+2}=0 \text { otherwise, }
\end{array}
$$

and $\lambda^{2}=\mu^{2}$.

Proposition 3.3. Let $M$ be an $m$-dimensional compact submanifold in the unit sphere $S^{m+p}$. If

$$
\kappa-m\|\boldsymbol{\Phi}\|^{2}-\frac{m}{4(m-1)}\|\tilde{\boldsymbol{A}}+\check{\boldsymbol{B}}\|^{2} \geq \frac{1}{m^{2}}\left(m-2+\frac{1}{2}(m-1) \operatorname{sgn}(p-1)\right),
$$

then

$$
\kappa-m\|\boldsymbol{\Phi}\|^{2}-\frac{m}{4(m-1)}\|\tilde{\boldsymbol{A}}+\check{\boldsymbol{B}}\|^{2}=\frac{1}{m^{2}}\left(m-2+\frac{1}{2}(m-1) \operatorname{sgn}(p-1)\right),
$$

and only the following two cases happen:

(i) $p=1$,

$$
\boldsymbol{\Phi}=0, \quad B_{i j}^{m+1}=\left\{\begin{aligned}
(\sqrt{m-1} / m) \sqrt{(m-k) / k} \delta_{i j} & \text { if } 1 \leq i \leq k, \\
-(\sqrt{m-1} / m) \sqrt{k /(m-k)} \delta_{i j} & \text { if } k+1 \leq i \leq m,
\end{aligned}\right.
$$

and

$$
A_{i j}=\left\{\begin{array}{lrr}
\frac{m-1}{m^{2}} \cdot \frac{3 k m-k^{2}-m^{2}}{2 k(m-k)} \delta_{i j} & \text { if } & 1 \leq i \leq k, \\
\frac{m-1}{m^{2}} \cdot \frac{m^{2}-k^{2}-k m}{2 k(m-k)} \delta_{i j} & \text { if } k+1 \leq i \leq m .
\end{array}\right.
$$

(ii) $p=2, m=2$, and

$$
\boldsymbol{\Phi}=0, \quad \boldsymbol{A}=\frac{3}{16} \boldsymbol{g}, \quad B^{3}=\frac{\sqrt{2}}{4}\left(\begin{array}{ll}
0 & 1 \\
1 & 0
\end{array}\right), \quad B^{4}=\frac{\sqrt{2}}{4}\left(\begin{array}{rr}
1 & 0 \\
0 & -1
\end{array}\right) .
$$


Proof. We consider two traceless tensors

$$
\check{\boldsymbol{B}}=\frac{m-1}{m^{2}} \boldsymbol{g}-B^{\alpha} B^{\alpha} \quad \text { and } \quad \tilde{\boldsymbol{A}}=\boldsymbol{A}-\frac{1}{m} \operatorname{tr}(\boldsymbol{A}) \boldsymbol{g} .
$$

Noting that

$$
\begin{aligned}
m \cdot \operatorname{tr}\left(B^{\alpha} B^{\alpha} \boldsymbol{A}\right) & =\frac{m-1}{m} \operatorname{tr}(\boldsymbol{A})-m \operatorname{tr}(\tilde{\boldsymbol{A}} \check{\boldsymbol{B}}) \\
& =\frac{m-1}{m} \operatorname{tr}(\boldsymbol{A})-\frac{1}{4} m\left(\|\tilde{\boldsymbol{A}}+\check{\boldsymbol{B}}\|^{2}-\|\tilde{\boldsymbol{A}}-\check{\boldsymbol{B}}\|^{2}\right) \\
& \geq \frac{m-1}{m} \operatorname{tr}(\boldsymbol{A})-\frac{1}{4} m\|\tilde{\boldsymbol{A}}+\check{\boldsymbol{B}}\|^{2},
\end{aligned}
$$

we see that the equality holds if and only if $\check{\boldsymbol{B}}=\tilde{\boldsymbol{A}}$. From (3-1), (3-3) and (3-4), we have

$$
\begin{aligned}
& 0 \geq \int_{M}\left(\|\nabla \boldsymbol{B}\|^{2}-m(m-1)\|\boldsymbol{\Phi}\|^{2}-\left(1+\frac{1}{2} \operatorname{sgn}(p-1)\right)\|\boldsymbol{B}\|^{4}\right. \\
& \left.+\frac{m-1}{m} \operatorname{tr}(\boldsymbol{A})+m \operatorname{tr}\left(B^{\alpha} B^{\alpha} \boldsymbol{A}\right)\right) d M \\
& \geq \int_{M}\left(\|\nabla \boldsymbol{B}\|^{2}-m(m-1)\|\boldsymbol{\Phi}\|^{2}\right. \\
& \left.-\frac{1}{4} m\|\tilde{\boldsymbol{A}}+\check{\boldsymbol{B}}\|^{2}-\left(1+\frac{1}{2} \operatorname{sgn}(p-1)\right)\|\boldsymbol{B}\|^{4}+2 \frac{m-1}{m} \operatorname{tr}(\boldsymbol{A})\right) d M \\
& \geq \frac{m-1}{m} \int_{M}\left(2 \operatorname{tr}(\boldsymbol{A})-m^{2}\|\boldsymbol{\Phi}\|^{2}-\frac{m^{2}}{4(m-1)}\|\tilde{\boldsymbol{A}}+\check{\boldsymbol{B}}\|^{2}\right. \\
& \left.-\left(1+\frac{1}{2} \operatorname{sgn}(p-1)\right) \frac{m-1}{m}\right) d M .
\end{aligned}
$$

Thus, if (3-6) holds all over $M$, then from (2-8) we know that (3-7) holds all over $M$ and $\nabla \boldsymbol{B}=0$. The latter implies $\boldsymbol{\Phi}=0$. At the same time, equality holds in (3-11), and so we have

$$
\boldsymbol{A}=-B^{\alpha} B^{\alpha}+\frac{1}{m}\left(\operatorname{tr}(A)+\frac{m-1}{m}\right) \boldsymbol{g} .
$$

Noting that the equality holds in (3-5), from Lemma 3.2 we know that only the case $p=1$ or $p=2$ is possible. So we consider the cases $p=1$ and $p=2$, separately.

Case: $p=1$. Let $B=B^{m+1}$. We choose our local frame field so that $B_{i j}=B_{i i} \delta_{i j}$. (Here and elsewhere in this case, repeated indexes such as those in $B_{i i}$ are not summed over.) Then from (3-12) we have $A_{i j}=A_{i i} \delta_{i j}$. Since $\nabla \boldsymbol{B}=0$ and $\boldsymbol{\Phi}=0$, from (2-4) we know that $B_{i i}$ is constant, $1 \leq i \leq m$, and

$$
0=B_{i j, k} \omega_{k}=d B_{i j}+B_{i k} \omega_{k j}+B_{k j} \omega_{k i}=\left(B_{i i}-B_{j j}\right) \omega_{i j},
$$


This shows that $\omega_{i j}=0$ if $B_{i i} \neq B_{j j}$. Hence

$$
-\frac{1}{2} R_{i j k l} \omega_{k} \wedge \omega_{l}=d \omega_{i j}-\omega_{i k} \wedge \omega_{k j}=0 .
$$

Then (3-13) and the Gauss Equation (2-5) implies that if $B_{i i} \neq B_{j j}$, then

$$
B_{i i} B_{j j}+A_{i i}+A_{j j}=0 .
$$

From (3-12), we have

$$
A_{i i}=-\left(B_{i i}\right)^{2}+\frac{1}{m}(\operatorname{tr}(A)+(m-1) / m) .
$$

Then (3-14) and (3-15) yield

$$
B_{i i} B_{j j}-\left(B_{i i}\right)^{2}-\left(B_{j j}\right)^{2}+2 t=0,
$$

where $m t=\operatorname{tr}(\boldsymbol{A})+(m-1) / m$. We will show that there are only two distinct $B_{i i}$. In fact, if three are distinct - say $B_{i i}, B_{j j}$ and $B_{k k}$ - then we have

$$
\begin{aligned}
B_{i i} B_{j j}-\left(B_{i i}\right)^{2}-\left(B_{j j}\right)^{2}+2 t & =0, \\
B_{i i} B_{k k}-\left(B_{i i}\right)^{2}-\left(B_{k k}\right)^{2}+2 t & =0, \\
B_{j j} B_{k k}-\left(B_{j j}\right)^{2}-\left(B_{k k}\right)^{2}+2 t & =0 .
\end{aligned}
$$

This implies

$$
\begin{aligned}
\left(B_{j j}-B_{k k}\right)\left(B_{i i}-\left(B_{j j}+B_{k k}\right)\right) & =0, \\
\left(B_{i i}-B_{k k}\right)\left(B_{j j}-\left(B_{i i}+B_{k k}\right)\right) & =0 \\
\left(B_{i i}-B_{j j}\right)\left(B_{k k}-\left(B_{i i}+B_{j j}\right)\right) & =0
\end{aligned}
$$

which yields

$$
\begin{aligned}
& B_{i i}-\left(B_{j j}+B_{k k}\right)=0, \\
& B_{j j}-\left(B_{i i}+B_{k k}\right)=0, \\
& B_{k k}-\left(B_{i i}+B_{j j}\right)=0 .
\end{aligned}
$$

These equations lead to $B_{i i}=0, B_{j j}=0$, and $B_{k k}=0$, a contradiction.

Thus we may assume $B_{11}=\cdots=B_{k k}=\lambda$ and $B_{k+1 k+1}=\cdots=B_{m m}=\mu$. We see that $\lambda, \mu, A_{i i}$ and $A_{j j}$ satisfy the following relations for $1 \leq i \leq k$ and $k+1 \leq j \leq m$ :

$$
\left\{\begin{array}{l}
\lambda \mu-\lambda^{2}-\mu^{2}+\frac{2}{m}(\operatorname{tr}(\boldsymbol{A})+(m-1) / m)=0, \\
k \lambda+(m-k) \mu=0, \\
k \lambda^{2}+(m-k) \mu^{2}=(m-1) / m, \\
A_{i i}=-\lambda^{2}+\frac{1}{m}(\operatorname{tr}(\boldsymbol{A})+(m-1) / m) \quad \text { for } 1 \leq i \leq k, \\
A_{j j}=-\mu^{2}+\frac{1}{m}(\operatorname{tr}(\boldsymbol{A})+(m-1) / m) \quad \text { for } k+1 \leq j \leq m, \\
\operatorname{tr}(\boldsymbol{A})=\sum_{l} A_{l l}=k A_{i i}+(m-k) A_{j j} .
\end{array}\right.
$$


From this system of equations, we get

$$
\begin{aligned}
\lambda & =\frac{\sqrt{m-1}}{m} \sqrt{\frac{m-k}{k}}, & \mu & =-\frac{\sqrt{m-1}}{m} \sqrt{\frac{k}{m-k}}, \\
A_{i i} & =\frac{m-1}{m^{2}} \cdot \frac{3 m k-k^{2}-m^{2}}{2 k(m-k)}, & A_{j j} & =\frac{m-1}{m^{2}} \cdot \frac{m^{2}-k^{2}-m k}{2 k(m-k)},
\end{aligned}
$$

where $1 \leq i \leq k$ and $k+1 \leq j \leq m$.

Case: $p=2$. In this case, $B^{m+1}=\lambda \tilde{B}^{m+1}$ and $B^{m+2}=\mu \tilde{B}^{m+2}$, where $\tilde{B}^{m+1}$ and $\tilde{B}^{m+2}$ are defined in Lemma 3.2 and $\lambda^{2}=\mu^{2}$. Noting that $2\left(\lambda^{2}+\mu^{2}\right)=\|\boldsymbol{B}\|^{2}=$ $(m-1) / m$, we have $2 \lambda=2 \mu=\sqrt{(m-1) / m}$. We get

$$
B^{m+1}=\frac{1}{2} \sqrt{\frac{m-1}{m}} \tilde{B}^{m+1} \quad \text { and } \quad B^{m+2}=\frac{1}{2} \sqrt{\frac{m-1}{m}} \tilde{B}^{m+2} .
$$

Since $B_{i j, k}^{\alpha}=0$, we have from (2-10)

$$
d B_{i j}^{\alpha}=-B_{k j}^{\alpha} \omega_{k i}-B_{i k}^{\alpha} \omega_{k j}-B_{i j}^{\beta} \omega_{\beta \alpha} .
$$

Setting $\alpha=m+1, i=1$ and $j \geq 3$, we see that by using (3-16) and (3-17) we get

$$
\omega_{2 j}=0 \quad \text { and } \quad j \geq 3 .
$$

Setting $\alpha=m+1, i=2$ and $j \geq 3$, we see from (3-16) and (3-17) that

$$
\omega_{1 j}=0 \quad \text { and } \quad j \geq 3 .
$$

Thus if $j \geq 3$, then

$$
0=d \omega_{1 j}=\omega_{1 k} \wedge \omega_{k j}-\left(A_{11}+A_{j j}\right) \omega^{1} \wedge \omega^{j}=-\left(A_{11}+A_{j j}\right) \omega^{1} \wedge \omega^{j} .
$$

Since $\omega^{1}, \ldots, \omega^{m}$ are orthonormal, $\omega^{1} \wedge \omega^{j}=0$ implies $\omega^{j}=0$ for $j \geq 3$. Thus this shows that $m=2$. Thus we have

$$
A=\frac{3}{16} g, \quad B^{3}=\frac{\sqrt{2}}{4}\left(\begin{array}{ll}
0 & 1 \\
1 & 0
\end{array}\right), \quad B^{4}=\frac{\sqrt{2}}{4}\left(\begin{array}{rr}
1 & 0 \\
0 & -1
\end{array}\right) .
$$

This completes the proof of Proposition 3.3.

Proof of Theorem 1.1. We first consider some examples.

Example 3.4. First, the Willmore torus $W_{k}^{m}=S^{k}(\sqrt{(m-k) / m}) \times S^{m-k}(\sqrt{k / m})$ in $S^{m+1}$. In [Guo et al. 2001], we proved that the Möbius invariants $\boldsymbol{\Phi}, \boldsymbol{A}$ and $\boldsymbol{B}$ of $W_{k}^{m}$ locally coincide with (3-8) and (3-9).

Example 3.5. Second, the Veronese surface in $S^{4}$. Let $(x, y, z)$ be the natural coordinate system in $R^{5}$. The mapping defined by $u^{1}=\frac{1}{\sqrt{3}} y z, u^{2}=\frac{1}{\sqrt{3}} z x, u^{3}=\frac{1}{\sqrt{3}} x y, u^{4}=\frac{1}{2 \sqrt{3}}\left(x^{2}-y^{2}\right), u^{5}=\frac{1}{6}\left(x^{2}+y^{2}-2 z^{2}\right)$ 
gives an immersion of $S^{2}(\sqrt{3})$ into $S^{4}$. We can choose the local frame field $\left\{e_{1}, e_{2}, e_{3}, e_{4}\right\}$ so that

$$
\left(h_{i j}^{3}\right)=\frac{1}{\sqrt{3}}\left(\begin{array}{ll}
0 & 1 \\
1 & 0
\end{array}\right) \quad \text { and } \quad\left(h_{i j}^{4}\right)=-\frac{1}{\sqrt{3}}\left(\begin{array}{cc}
1 & 0 \\
0 & -1
\end{array}\right) .
$$

Thus $H=0$ and $\rho^{2}=8 / 3$. Putting these into (1-1), (1-2) and (1-3), we have

$$
\boldsymbol{\Phi}=0, \quad \boldsymbol{A}=\frac{3}{16} \boldsymbol{g}, \quad B^{3}=\frac{\sqrt{2}}{4}\left(\begin{array}{ll}
0 & 1 \\
1 & 0
\end{array}\right), \quad B^{4}=\frac{\sqrt{2}}{4}\left(\begin{array}{cc}
1 & 0 \\
0 & -1
\end{array}\right) .
$$

It is known that if two submanifolds in $S^{m+p}$ have the same Möbius metric, Möbius second fundamental form, and Blaschke tensor, then they are locally Möbius equivalent. Since we assume $M$ is compact in Theorem 1.1, we complete the proof by comparing (3-8), (3-9) and (3-10) with Examples 3.4 and 3.5.

Proposition 3.6. Let $x: M \rightarrow S^{m+p}$ be an m-dimensional compact submanifold in the unit sphere $S^{m+p}$. Let $K(x)$ be the function that assigns to each point of $M$ the infinimum of the Möbius sectional curvatures of $M$ at that point. Then

$$
\int_{M}\left(2(m+1) K-m \kappa-1 / m-m(m+2)\|\boldsymbol{\Phi}\|^{2}-m\|\tilde{\boldsymbol{A}}\|\right) d M \leq 0 .
$$

In particular, if

$$
2(m+1) K-m \kappa-m(m+2)\|\boldsymbol{\Phi}\|^{2}-m\|\tilde{\boldsymbol{A}}\| \geq 1 / m,
$$

then

$$
2(m+1) K-m \kappa-m(m+2)\|\boldsymbol{\Phi}\|^{2}-m\|\tilde{\boldsymbol{A}}\|=1 / m,
$$

and

$$
K=\kappa=\frac{1}{m(m+2)}, \quad \boldsymbol{\Phi}=0, \quad \boldsymbol{A}=\frac{1}{m} \operatorname{tr}(\boldsymbol{A}) \boldsymbol{g} .
$$

Proof. In the proof, we will use Yau's technique; see [Yau 1975; Itoh 1975; 1978]. By defining

$$
\left\|R^{\perp}\right\|^{2}=\operatorname{tr}\left(\left(B^{\alpha} B^{\beta}-B^{\beta} B^{\alpha}\right)\left(B^{\beta} B^{\alpha}-B^{\alpha} B^{\beta}\right)\right)
$$

and noting that $B_{j l}^{\alpha} B_{l k}^{\beta} R_{\beta \alpha j k}=-\frac{1}{2}\left\|R^{\perp}\right\|^{2}$, from (3-2) and (3-3) we have

$$
\begin{array}{r}
0=\int_{M}\left(\|\nabla \boldsymbol{B}\|^{2}-m(m-1)\|\boldsymbol{\Phi}\|^{2}+(1+a)\left(B_{k j}^{\alpha} B_{m l}^{\alpha} R_{m k j l}+B_{j k}^{\alpha} B_{k m}^{\alpha} R_{l j l m}\right)\right. \\
+a \operatorname{tr}\left(B^{\alpha} B^{\beta}\right) \operatorname{tr}\left(B^{\alpha} B^{\beta}\right)-a(2(m-1) / m) \operatorname{tr}(\boldsymbol{A}) \\
\left.-a m \operatorname{tr}\left(B^{\alpha} B^{\alpha} \tilde{\boldsymbol{A}}\right)-\frac{1}{2}(1-a)\left\|R^{\perp}\right\|^{2}\right) d M
\end{array}
$$

where $a$ is a real number. 
For each fixed $\bar{\alpha}$, let $\alpha_{i}$ be the eigenvalues of the matrix $\left(B_{i j}^{\bar{\alpha}}\right)$. By the definition of $K$, we have

$$
\begin{aligned}
\left(B_{k j}^{\bar{\alpha}} B_{m l}^{\bar{\alpha}} R_{m k j l}+B_{j k}^{\bar{\alpha}} B_{k m}^{\bar{\alpha}} R_{l j l m}\right) & =\frac{1}{2}\left(\alpha_{i}-\alpha_{j}\right)\left(\alpha_{i}-\alpha_{j}\right) R_{i j i j} \\
& \geq \frac{1}{2}\left(\alpha_{i}-\alpha_{j}\right)\left(\alpha_{i}-\alpha_{j}\right) K \\
& =m K B_{i j}^{\bar{\alpha}} B_{i j}^{\bar{\alpha}} .
\end{aligned}
$$

The following equality is called the Lincoln equality (also see [Itoh 1978]):

$$
\left\|R^{\perp}\right\|^{2} \leq m \operatorname{tr}\left(B^{\alpha} B^{\beta}\right) \operatorname{tr}\left(B^{\alpha} B^{\beta}\right),
$$

where the equality implies

$$
\left(B_{i k}^{\alpha} B_{j l}^{\alpha}-B_{i l}^{\alpha} B_{j k}^{\alpha}\right)=-\frac{1}{m^{2}}\left(\delta_{i k} \delta_{j l}-\delta_{i l} \delta_{j k}\right) .
$$

Hence, for $a \geq 0$, by putting (3-23) and (3-24) into (3-22), we have

$$
\begin{aligned}
& 0 \geq \int_{M}\left(\|\nabla \boldsymbol{B}\|^{2}-m(m-1)\|\boldsymbol{\Phi}\|^{2}+(1+a)(m-1) K\right. \\
&-a(2((m-1) / m) \operatorname{tr}(A)\left.+m \operatorname{tr}\left(B^{\alpha} B^{\alpha} \tilde{\boldsymbol{A}}\right)\right) \\
&\left.+\left(a / m-\frac{1}{2}(1-a)\right)\left\|R^{\perp}\right\|^{2}\right) d M .
\end{aligned}
$$

By Schwarz's inequality, we have

$$
\operatorname{tr}\left(\tilde{\boldsymbol{A}} B^{\alpha} B^{\alpha}\right) \leq\|\tilde{\boldsymbol{A}}\|\left\|B^{\alpha}\right\|^{2},
$$

where the equality holds when there exist real numbers $a^{\alpha}$ such that

$$
A=a^{\alpha}\left(B^{\alpha}\right)^{2} \text { for } m+1 \leq \alpha \leq m+p .
$$

Now by taking $a=m /(m+2)$ in (3-25), and (2-8) and (3-27), we have

$$
\begin{aligned}
0 \geq \int_{M}\left(\|\nabla \boldsymbol{B}\|^{2}-m(m-1)\right. & \|\boldsymbol{\Phi}\|^{2} \\
& \left.+\frac{m-1}{m+2}\left(2(m+1) K-m \kappa-\frac{1}{m}-m\|\tilde{\boldsymbol{A}}\|\right)\right) d M
\end{aligned}
$$

Thus we get (3-18). If (3-19) holds, then we have (3-20), $\nabla \boldsymbol{B}=0$ and the equality holding in (3-6). These implies $\boldsymbol{\Phi}=0$ and $\tilde{\boldsymbol{A}}=0$. From (2-2), we get $A_{i j}=\lambda \delta_{i j}$, $\lambda=\operatorname{tr}(A) / m=$ constant. Now (3-25) holds since equality holds in (3-24). Hence, from (2-5) we know that the Möbius sectional curvature is constant. This implies that $K=\kappa$. Substituting these into (3-20), we have $K=\kappa=1 /(m(m+2))$.

Proof of Theorem 1.2. Under the assumptions of Theorem 1.2, from Proposition 3.6 we have $\boldsymbol{\Phi}=0, \quad \tilde{\boldsymbol{A}}=0$ and $\operatorname{tr}(\boldsymbol{A})=\left(1+m^{2} \kappa\right) /(2 m)>0$. According to the classification theorem on Möbius isotropic submanifolds, proved by Liu, Wang, and Zhao [2001], $M$ is Möbius equivalent to a minimal (Euclidean) submanifold $\bar{M}$ with constant Euclidean scalar curvature in $S^{m+p}$. Since $\bar{M}$ shares same $\boldsymbol{A}, \boldsymbol{B}$ 
and $K$ with $M$, we can use the same symbols to denote the Möbius quantities of $\bar{M}$. Now we can compute the Euclidean sectional curvature $K_{E}$ of $\bar{M}$. In fact, since $\bar{M}$ is Euclidean minimal and its Euclidean scalar curvature is constant, we have $\rho^{2}=$ constant. From (1-2), we have $A_{i j}=\frac{1}{2} \rho^{-2} \delta_{i j}$. From $g=\rho^{2} d x \cdot d x$, we have

$$
K=\rho^{-2} K_{E},
$$

Noting that $\rho^{-2}=2 / m \operatorname{tr}(\boldsymbol{A})$ and $\operatorname{tr}(\boldsymbol{A})=\left(1+m^{2} \kappa\right) /(2 m)$, we have

$$
\rho^{-2}=1 / m^{2}+\kappa \text {. }
$$

From Proposition 3.6 we have $\kappa=K=1 /(m(m+2))$. Hence, from (3-29) and (3-30) we get $K_{E}=m /(2(m+1))$. Since $\bar{M}$ is a Euclidean minimal submanifold with sectional curvature $K_{E}$ in $S^{m+p}$, Itoh's theorem [1978] shows that $\bar{M}$ is isometric to the Veronese submanifold $S^{m}(\sqrt{2(m+1) / m})$. Thus $M$ is Möbius equivalent to Veronese submanifold

$$
x_{m}: S^{m}(\sqrt{2(m+1) / m}) \rightarrow S^{m+p},
$$

where $p=\frac{1}{2}(m-1)(m+2)$. This proves Theorem 1.2.

Proof of Corollary 1.3. We assume $c \geq 1 /(m(m+2))$. Under the assumptions of Corollary 1.3, we have $\Phi=0, \kappa=c$ and $K=c \geq 1 /(m(m+2))$. Writing $A_{i j}=\lambda_{i} \delta_{i j}$, from (2-2) and (2-12), we have

$$
\frac{1}{2} \Delta\|\tilde{A}\|^{2}=A_{i j k} A_{i j k}+\frac{1}{2} \sum_{i, j}\left(\lambda_{i}-\lambda_{j}\right)^{2} R_{i j i j},
$$

where $\tilde{A}_{i j}=A_{i j}-(\operatorname{tr} A / m) \delta_{i j}$ and $\|\tilde{A}\|^{2}=\tilde{A}_{i j} \tilde{A}_{i j}$. Thus, from (3-31) we have

$$
\tilde{A}=0 .
$$

In this case, (1-6) is equivalent to $c \geq 1 /(m(m+2))$. Thus from Theorem $1.2, M$ is Möbius equivalent to Veronese submanifold $S^{m}(\sqrt{2(m+1) / m})$.

\section{Möbius sectional curvature pinching theorems}

Theorem 4.1. Let $x: M \rightarrow S^{m+p}$ be an m-dimensional compact submanifold in the unit sphere $S^{m+p}$. Let $K(x)$ be the function that assigns to each point of $M$ the infinimum of the Möbius sectional curvatures of $M$ at that point. Then

$$
\int_{M}\left(K-\frac{m-1}{2 m^{2}}\left(1-\frac{1}{p}+\frac{1}{2} \operatorname{sgn}(p-1)\right)-m\|\boldsymbol{\Phi}\|^{2}-2\|\tilde{\boldsymbol{A}}\|\right) d M \leq 0 .
$$

In particular, if

$$
K \geq \frac{m-1}{2 m^{2}}\left(1-\frac{1}{p}+\frac{1}{2} \operatorname{sgn}(p-1)\right)+m\|\boldsymbol{\Phi}\|^{2}+2\|\tilde{\boldsymbol{A}}\|,
$$


then either

(i) $p=1, K=0$ and $M$ is Möbius equivalent to the Clifford minimal torus $M_{k}^{m}=S^{k}(\sqrt{k /(m-k)}) \times S^{m-k}(\sqrt{(m-k) / m})$ in $S^{m+1}$, or

(ii) $m=2, p=2, K=1 / 8$ and $M$ is Möbius equivalent to a Veronese surface in $S^{4}$.

Theorem 4.2. Let $x: M \rightarrow S^{m+p}$ for $p \geq 2$ be an m-dimensional compact submanifold in the unit sphere $S^{m+p}$. Let $K(x)$ be the function that assigns to each point of $M$ the infinimum of the Möbius sectional curvatures of $M$ at that point. Then

$$
\int_{M}\left(K-\frac{(m-1)(3 p-2)}{4 p m^{2}}-m\|\boldsymbol{\Phi}\|^{2}\right) d M \leq 0 .
$$

In particular, if

$$
K \geq(m-1)(3 p-2) /\left(4 p m^{2}\right)+m\|\boldsymbol{\Phi}\|^{2},
$$

then $m=2, \quad p=2, K=1 / 8$ and $M$ is Möbius equivalent to a Veronese surface in $S^{4}$.

Proof of Theorem 4.1. Choosing $a=1$ in (3-22), using (3-23) and (2-8) we have

$$
\begin{aligned}
& 0=\int_{M}\left(\|\nabla \boldsymbol{B}\|^{2}-m(m-1)\|\boldsymbol{\Phi}\|^{2}\right. \\
& +2\left(B_{k j}^{\alpha} B_{m l}^{\alpha} R_{m k j l}+B_{j k}^{\alpha} B_{k m}^{\alpha} R_{l j l m}\right) \\
& \left.+\operatorname{tr}\left(B^{\alpha} B^{\beta}\right) \operatorname{tr}\left(B^{\alpha} B^{\beta}\right)-\frac{1}{m} 2(m-1) \operatorname{tr}(\boldsymbol{A})-m \operatorname{tr}\left(B^{\alpha} B^{\alpha} \tilde{\boldsymbol{A}}\right)\right) d M \\
& \geq \int_{M}\left(\|\nabla \boldsymbol{B}\|^{2}-m(m-1)\|\boldsymbol{\Phi}\|^{2}+2(m-1) K\right. \\
& \left.+\frac{1}{p}\left(\frac{m-1}{m}\right)^{2}-\frac{2(m-1)}{m}\left(\frac{1}{2 m}\left(1+m^{2} \kappa\right)\right)-(m-1)\|\tilde{\boldsymbol{A}}\|\right) d M \\
& =\int_{M}\left(\|\nabla \boldsymbol{B}\|^{2}-m(m-1)\|\boldsymbol{\Phi}\|^{2}+2(m-1) K\right. \\
& \left.+\frac{m-1}{m^{2}}\left(\frac{m-1}{p}-1\right)-(m-1) \kappa-(m-1)\|\tilde{\boldsymbol{A}}\|\right) d M .
\end{aligned}
$$

Using (1-4), we get (4-1). If (4-2) holds, we have $\|\nabla \boldsymbol{B}\|=0$ on $M$. The conclusion of Theorem 4.1 follows in the same way as in the proof of Theorem 1.1.

Proof of Theorem 4.2. Choosing $a=0$ in (3-22) and using (3-23) and (2-8), we have

$$
0 \geq \int_{M}\left(\|\nabla \boldsymbol{B}\|^{2}-m(m-1)\|\boldsymbol{\Phi}\|^{2}+(m-1) K-\frac{1}{2}\left\|R^{\perp}\right\|^{2}\right) d M
$$


Combining (4-5), (3-21) with Lemma 3.2, we have

$$
\begin{aligned}
0 \geq \int_{M}\left(\|\nabla \boldsymbol{B}\|^{2}-m(m-1)\|\boldsymbol{\Phi}\|^{2}\right. & +(m-1) K \\
& -\frac{1}{2}\left(\frac{3}{2}\|\boldsymbol{B}\|^{4}-\operatorname{tr}\left(B^{\alpha} B^{\beta} B^{\alpha} B^{\beta}\right)\right) d v \\
\geq \int_{M}\left(\|\nabla \boldsymbol{B}\|^{2}-m(m-1)\|\boldsymbol{\Phi}\|^{2}\right. & +(m-1) K \\
& \left.-\frac{3}{4}\left(\frac{m-1}{m}\right)^{2}+\frac{1}{2 p}\left(\frac{m-1}{m}\right)^{2}\right) d M .
\end{aligned}
$$

Thus we get (4-3). If (4-4) holds on $M$, then we have $\|\nabla \mathbf{B}\|=0$ on $M$. The conclusion of Theorem 4.2 then follows as for Theorem 1.1.

\section{References}

[Akivis and Goldberg 1996] M. A. Akivis and V. V. Goldberg, Conformal differential geometry and its generalizations, Wiley, New York, 1996. MR 98a:53023 Zbl 0863.53002

[Akivis and Goldberg 1997] M. A. Akivis and V. V. Goldberg, "A conformal differential invariant and the conformal rigidity of hypersurfaces", Proc. Amer. Math. Soc. 125:8 (1997), 2415-2424. MR 97j:53017 Zbl 0887.53030

[Blaschke 1929] W. Blaschke, Vorlesungen über Differentialgeometrie und geometrische Grundlagen von Einsteins Relativitätstheorie, III: Differentialgeometrie der Kreise und Kugeln, Springer, Berlin, 1929. JFM 55.0422.01

[Chen 1984] B.-Y. Chen, Total mean curvature and submanifolds of finite type, Series in Pure Mathematics 1, World Scientific, Singapore, 1984. MR 86b:53053 Zbl 0537.53049

[Chern et al. 1970] S. S. Chern, M. do Carmo, and S. Kobayashi, "Minimal submanifolds of a sphere with second fundamental form of constant length", pp. 59-75 in Functional analysis and related fields (Chicago, 1968), edited by F. E. Browder, Springer, New York, 1970. MR 42 \#8424 Zbl 0216.44001

[Guo and Guo 2006] Z. Guo and K. Guo, "Möbius characterization of some submanifolds in the unit sphere”, Results Math. 49:3-4 (2006), 219-236. MR 2007h:53082 Zbl 1128.53011

[Guo et al. 2001] Z. Guo, H. Li, and C. Wang, "The second variational formula for Willmore submanifolds in $S^{n}$ ”, Results Math. 40:1-4 (2001), 205-225. MR 2003b:53065 Zbl 01698602

[Hu and Li 2003] Z. Hu and H. Li, "Submanifolds with constant Möbius scalar curvature in $S^{n}$ ", Manuscripta Math. 111:3 (2003), 287-302. MR 2004c:53080 Zbl 1041.53007

[Hu and $\mathrm{Li}$ 2004] Z. Hu and H. Li, "Classification of hypersurfaces with parallel Möbius second fundamental form in $S^{n+1}$, Sci. China Ser. A 47:3 (2004), 417-430. MR 2005c:53066 Zbl 1082.53016

[Itoh 1975] T. Itoh, “On Veronese manifolds”, J. Math. Soc. Japan 27:3 (1975), 497-506. MR 52 \#4190 Zbl 0303.53055

[Itoh 1978] T. Itoh, “Addendum to [Itoh 1975]”, J. Math. Soc. Japan 30:1 (1978), 73-74. MR 58 \#24135 Zbl 0363.53025

[Lawson 1969] H. B. Lawson, Jr., "Local rigidity theorems for minimal hypersurfaces", Ann. of Math. (2) 89 (1969), 187-197. MR 38 \#6505 Zbl 0174.24901

[Li 2001] H. Li, "Willmore hypersurfaces in a sphere", Asian J. Math. 5:2 (2001), 365-377. MR 2003e:53077 Zbl 1025.53031 
[Li 2002] H. Li, "Willmore submanifolds in a sphere", Math. Res. Lett. 9:5-6 (2002), 771-790. MR 2003j:53092 Zbl 1056.53040

[Li and Li 1992] A.-M. Li and J.-M. Li, "An intrinsic rigidity theorem for minimal submanifolds in a sphere”, Arch. Math. (Basel) 58:6 (1992), 582-594. MR 93b:53050 Zbl 0731.53056

[Li and Wang 2003a] H. Li and C. Wang, "Möbius geometry of hypersurfaces with constant mean curvature and scalar curvature", Manuscripta Math. 112:1 (2003), 1-13. MR 2004e:53092 Zbl 1041.53008

[Li and Wang 2003b] H. Z. Li and C. P. Wang, "Surfaces with vanishing Möbius form in $S^{n}$ ", Acta Math. Sin. (Engl. Ser.) 19:4 (2003), 671-678. MR 2004j:53076 Zbl 1078.53012

[Li et al. 2001] H. Li, C. Wang, and F. Wu, "A Möbius characterization of Veronese surfaces in $S^{n}$ ", Math. Ann. 319:4 (2001), 707-714. MR 2002b:53098 Zbl 1031.53086

[Liu et al. 2001] H. Liu, C. Wang, and G. Zhao, "Möbius isotropic submanifolds in $S^{n}$ ", Tohoku Math. J. (2) 53:4 (2001), 553-569. MR 2002f:53096 Zbl 1014.53010

[Pedit and Willmore 1988] F. J. Pedit and T. J. Willmore, "Conformal geometry", Atti Sem. Mat. Fis. Univ. Modena 36:2 (1988), 237-245. MR 89m:53107 Zbl 0665.53048

[Simons 1968] J. Simons, "Minimal varieties in Riemannian manifolds", Ann. of Math. (2) 88 (1968), 62-105. MR 38 \#1617 Zbl 0181.49702

[Wang 1998] C. Wang, "Möbius geometry of submanifolds in $S^{n}$ ”, Manuscripta Math. 96:4 (1998), 517-534. MR 2000a:53019 Zbl 0912.53012

[Yau 1975] S. T. Yau, "Submanifolds with constant mean curvature, II”, Amer. J. Math. 97 (1975), 76-100. MR 51 \#6670 Zbl 0304.53042

Received January 8, 2009.

\section{ZHEN GUO}

DEPARTMENT OF MATHEMATICS

YUNNAN NORMAL UNIVERSITY

KUNMING 650092

CHINA

gzh2001y@yahoo.com

\section{HAIZHONG LI}

DEPARTMENT OF MATHEMATICS SCIENCES

TSINGHUA UNIVERSITY

BEIJING 100084

CHINA

hli@math.tsinghua.edu.cn

CHANGPING WANG

Department of Mathematics

PEKING UNIVERSITY

BEIJING 100871

ChINA

wangcp@pku.edu.cn 